\title{
VEGF-C and podoplanin, as biomarkers of sepsis. An experimental study
}

\author{
Emoke Almasy ${ }^{1}$, Janos Szederjesi ${ }^{1 *}$, Bianca-Liana Grigorescu ${ }^{2}$, Simona Gurzu $^{3}$, \\ Alexandra Lazar ${ }^{1}$, Gergo Raduly ${ }^{4}$, Adrian Man ${ }^{5}$, Madalina Oprica ${ }^{6}$, Irina \\ Saplacan $^{1}$, Sanda-Maria Copotoiu ${ }^{7}$
}

1. Anesthesiology and Intensive Care, George Emil Palade University of Medicine, Pharmacy, Science, and Technology of Targu Mures, Romania

2. Pathophysiology, George Emil Palade University of Medicine, Pharmacy, Science, and Technology of Targu Mures, Romania

3. Pathology, George Emil Palade University of Medicine, Pharmacy, Science, and Technology of Targu Mures, Romania

4. Anatomy and Embryology, George Emil Palade University of Medicine, Pharmacy, Science, and Technology of Targu Mures, Romania

5. Microbiology, George Emil Palade University of Medicine, Pharmacy, Science, and Technology of Targu Mures, Romania

6. Humoral Immunology Laboratory Center for Advanced Medical and Pharmaceutical Research, George Emil Palade University of Medicine, Pharmacy, Science, and Technology of Targu Mures,

Romania

7. Anesthesiology and Intensive Care Clinic, George Emil Palade University of Medicine, Pharmacy, Science, and Technology of Targu Mures, Romania

\begin{abstract}
Background: Sepsis is the leading cause of morbidity and mortality in intensive care units. This study explored the possible role of vascular endothelial growth factor-C (VEGF-C) and podoplanin (PDPN) in sepsis. Methods: 22 Wistar rats were divided into three groups: two experimental (Group $A$ and B, $n=8 / 8$ ) and a control (Group $C$, $n=6)$. Sepsis was induced with intraperitoneal injection of ESBL (extended-spectrum beta-lactamases)-producing E-coli live bacteria for group A and with lipopolysaccharide for group B. Sterile saline solution was injected for
\end{abstract}

\footnotetext{
* Corresponding author: Janos Szederjesi, Anesthesiology and Intensive Care, George Emil Palade University of Medicine, Pharmacy, Science, and Technology of Targu Mures, Romania. E-mail: yangzi37@gmail.com
} 
group C. Blood samples were collected after 24 hours to determine the serum level of VEGF-C, and PDPN expression was examined in liver, kidney, and lung tissues. Bacteremia was assessed for group A. Results: Higher serum levels of VEGF-C were found in Group $A$ vs $C(p=0.05)$ and group $B$ vs. $C(p=0.004)$, respectively.VEGF-C was also increased in animals with negative- vs. positive blood cultures from group $A(p=0.04)$ and from group $B$ vs. those with positive blood cultures from group A ( $p=0.03)$. High intensity of PDPN tissue expression was observed in the pulmonary alveolocytes from Group $A$ and epithelium of the proximal renal tubules in groups $B$ and $C$, compared to group A. Conclusions: Circulating VEGF-C can be succesfuly used as a biomarker of sepsis with negative blood cultures and high risk of renal failure, whereas PDPN seems to exert a protective role against lung injuries in live bacteria-induced sepsis.

Keywords: podoplanin, vascular endothelial growth factor, sepsis, lipopolysaccharide

Received: $1^{\text {st }}$ September 2021; Accepted: 27 $7^{\text {th }}$ September 2021; Published: $1^{\text {st }}$ October 2021

\section{Introduction}

Sepsis, a major cause of death amongst critically ill patients, is the leading cause of morbidity and mortality in intensive care units (ICUs) $(1,2)$. Despite advances in antimicrobial therapy, sepsis remains a major public health problem due to its onset, evolution and outcome. Over 900,000 people are affected every year in the United States, with an incidence of 535 cases per 100,000 person-years. Although there are standardized therapeutic protocols, 270,000 deaths are reported annually in the United States, with a mortality rate between $20 \%$ and $36 \%$ (3-5).

Despite the fact that a variety of biomarkers are used in daily practice o establish the diagnosis and severity of sepsis, none have high enough specificity and sensitivity to distinguish an inflammatory syndrome of sepsis. No specific biomarkers are used in daily practice to diagnose sepsis with negative blood cultures (6-11). This opens up new research opportunities for early diagnosis of septic patients.

Vascular endothelial growth factor (VEGF) family of proteins includes five different polypeptides: placenta growth factor (PIGF), VEGF-A, VEGF-B, VEGF-C, VEGF-D (12-15). Their function depends upon binding to type $\mathrm{V}$ receptor tyrosine kinases. If VEGF-A and -B and their receptors (VEGF-R1 and -R2) are involved in angiogenesis, VEGF-C and its receptor- VEGF-R3, modulate lymphangiogenesis
(15-20). It was also postulated that VEGF-A/ VEGF-R1 and VEGF-B/-R2 are secreted by alveolar cells under hypoxic and septic conditions and induce injuries of the endothelial-alveolar barrier but few data are known about the role of VEGF-C and its receptor VEGF-R3 in modulating septic process (16).

Podoplanin (PDPN) is a transmembrane protein involved in embryogenesis and organ development, but also cell motility, carcinogenesis and metastasis. It is expressed on a variety of epithelial surfaces in multiple tissues, including brain, heart, lungs, kidney, osteoblasts, and lymphoid organs (17-19). The name of this protein is derived from its expression in kidney podocytes (20). It has been suggested that PDPN might have a role in sepsis but the pathological mechanism is far to be understood.

As PDPN was postulated to stimulate lymphangiogenesis and, as consequence, the inflammatory process and tissue edema, the aim of this study was to explore the possible role of the inflammatory storm, during sepsis, in inducing lymphatic flow disorders $(21,22)$. For this reason, tissue expression of PDPN was examined in association with the serum level of VEGF-C, in live-bacteria- vs. lipopolysaccharide (LPS)-induced sepsis. In order to assess the involvement of different bacterial components in the evolution of septic syndrome, either LPS or live bacteria were used to trigger the immune response 
in rats, by LPS alone, or by LPS as part of live bacteria aside other bacterial virulence factors.

\section{Materials and Methods}

This was an experimental study approved by the Ethics Committee of George Emil Palade University of Medicine, Pharmacy, Science and Technology of Targu Mures, Romania. The study comprised 22 adult male Wistar rats, aged 12 weeks, weighing $250 \mathrm{~g} \pm 20 \mathrm{~g}$, which were randomly divided into three groups: two experimental groups (Group A and Group B, $n=8$ for each group) and a control group (Group $C, n=6$ ). Two weeks prior the experiment the animals were acclimatized with the standard laboratory conditions which consisted of ad libitum food and water, $22^{\circ} \mathrm{C}$ environmental temperature and and a 12 hour light/dark cycle.

On the experimental day the rats were fasted four hours prior induction of general anesthesia with $5 \%$ Isoflurane in oxygen, $2 \mathrm{~L} / \mathrm{min}$ fresh gas flow rate, shifting to face mask with $2.5 \%$ Isoflurane in oxygen, $2 \mathrm{~L} / \mathrm{min}$ fresh gas flow rate. Sepsis was induced by intraperitoneal injection of pathogens, as follows:

Group A was injected with $0.7 \mathrm{ml}$ of $1.5 \times 10^{8}$ $\mathrm{CFU} / \mathrm{ml}$ of ESBL-producing $E$. coli live bacteria (clinical isolate, as identified by Vitek2 Compact automated system (bioMérieux SA, France) and by ROSCO Neo-Sensitabs tablet synergy tests (Rosco Diagnostica, Denmark)).

Group B was injected with $1 \mathrm{ml}$ ultrapure lipopolysaccharide (LPS) from Heat-Killed E. coli O111:B4, 107 CFU/ml (InvivoGen, USA).

Group $\mathrm{C}$ - control group, injected with $1 \mathrm{ml}$ of sterile saline solution.

Following anesthetic recovery, the animals' activity and respiratory rate were monitored and free access to water and food was allowed.

After 24 hours, the anesthesia with the afore-mentioned protocol was repeated and blood was harvested from the abdominal aorta.
The blood samples for serum VEGF-C levels (for all groups) and blood culture (for groups A and C) were collected when the animals were presenting clinical signs of deep anesthesia (lack of response to pain stimulus, shallow breath). Following blood harvesting the rats were sacrificed by exsanguination and tissue samples from liver, kidney and lungs were collected for further histopathological assessment.

\section{Detection of VEGF-C serum levels}

The blood samples for VEGF-C were collected on clot activator blood tubes $(5 \mathrm{ml})$ and left to rest for 30 minutes. The serum was separated by centrifugation for 5 minutes at 3500 rotations per minute and stored at $-20{ }^{\circ} \mathrm{C}$. Serum levels of VEGF-C were determined using VGEF-C enzyme-linked immunosorbent assay (ELISA) kit, (PromoCell GmbH, Germany) in a DYNEX DSX AUTOMATED ELISA SYSTEM analyzer. The VEGF-C detection limit of the kit was 27 $\mathrm{pg} / \mathrm{ml}$.

\section{Blood culture}

The blood culture samples $(4 \mathrm{ml})$ were inoculated in pediatric BACT/ALERT ${ }^{\circledR}$ PF culture media tubes and processed using the BacT/Alert3D (bioMérieux, France) microbial identification system. Bacteria from the positive blood cultures were identified using standard culture and biochemical methods.

\section{Immunohistochemistry}

Tissue samples from liver, kidney and lungs were fixed in $4 \%$ buffered formalin and then embedded in paraffin. $5 \mu \mathrm{m}$ sections of formalin-fixed paraffin-embedded tissues were dewaxed and rehydrated using standard methods. Endogenous peroxidases were blocked with $3 \% \mathrm{H} 2 \mathrm{O} 2$. Antigens were retrieved by pressurized steam cooking in buffer solution (EDTA-based, pH 9). Sections were incubated with a biotin-conjugated mouse monoclonal anti-PDPN antibody (clone 
eBio8.1.1, ThermoFisher Scientific, USA), at a dilution of 1:50, for $60 \mathrm{~min}$. 3, 3'-diaminobenzidine (DAB) was used to develop the stains. Nuclei were counterstained with Mayer's hematoxylin solution.

To quantify the cytoplasmic expression of PDPN, a scoring system previously described in the literature was used (23). The definitions of the distribution scores, the intensity scores, and the conversion to a binary positive/negative score are shown in Table I.

\section{Statistical analysis}

Statistical Package for Social Sciences (SPSS version 17, Chicago, IL, USA) was used to analyze the data. VEGF-C levels were compared using the Mann-Whitney test. Differences in serum VEGF-C between animals with positive and negative blood cultures (group A) were assessed using the Unpaired t-test. The Kruskal Wallis nonparametric test was used for comparing the differences between groups. The significance level was set at $\alpha=0.05$.

\section{Results}

\section{Circulating VEGF-C}

Slightly higher serum VEGF-C was found in Group A compared to Group C ( $p=0.05$, Mann Whitney), but the difference between Group B and $\mathrm{C}$ was even highly significant, with higher values in Group B ( $p=0.004)$. The VEGF-C serum level did not differ between Group $A$ and $B$ $(p=0.18)$. (Fig. 1).

In Group A four of the eight blood cultures were negative (data no shown). The animals with negative blood cultures presented pulmonary abscesses at autopsy. In group A, serum VEGF-C was significantly increased $(p=0.04)$ in animals with negative than in those with positive cultures. It was also significantly increased in group $\mathrm{B} v s$. cases with positive cultures from group A $(p=0.03)$. No differences between group B and cases with negative cultures from group A was emphasized $(\mathrm{p}=0.58)$. All blood cultures from group $\mathrm{C}$ were negative, confirming the lack of infection (Table II).

Table I. The scoring system used for the quantification of the cytoplasmic expression of the PDPN

\begin{tabular}{|c|c|c|}
\hline Percentage of stained cells in the tissue core & \multicolumn{2}{|c|}{ Distribution Score } \\
\hline 0 & \multicolumn{2}{|c|}{0} \\
\hline $1-50$ & \multicolumn{2}{|c|}{1} \\
\hline $51-100$ & \multicolumn{2}{|c|}{2} \\
\hline Intensity of signal & \multicolumn{2}{|c|}{ Intensity Score } \\
\hline No signal & \multicolumn{2}{|c|}{0} \\
\hline Weak & \multicolumn{2}{|c|}{1} \\
\hline Moderate & \multicolumn{2}{|c|}{2} \\
\hline Marked & \multicolumn{2}{|c|}{3} \\
\hline Sum (Distribution score + Intensity Score) & Total score & Expression \\
\hline 0 & \multirow{2}{*}{0} & \multirow{3}{*}{ Negative } \\
\hline 1 & & \\
\hline 2 & 1 & \\
\hline 3 & 2 & \multirow{3}{*}{ Positive } \\
\hline 4 & \multirow{2}{*}{3} & \\
\hline 5 & & \\
\hline
\end{tabular}




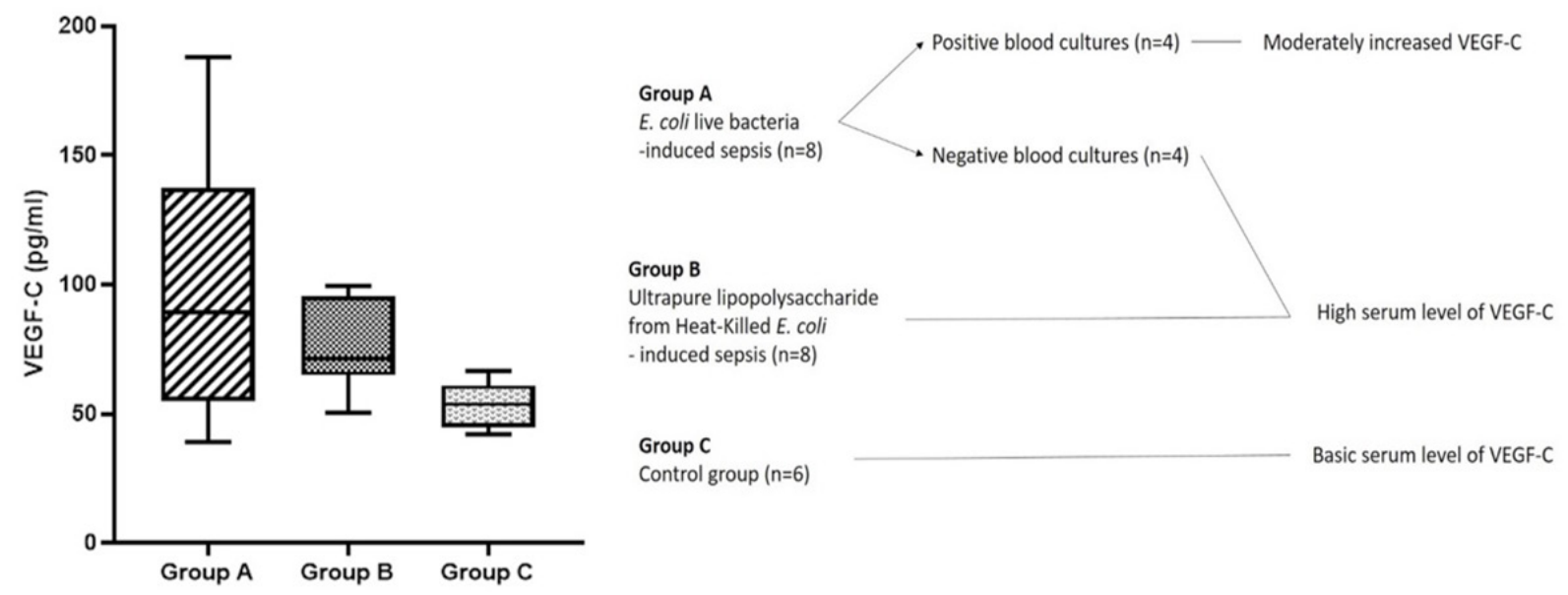

Fig. 1. Serum VEGF-C levels in sepsis $v s$. control group. The highest values are shown for sepsis with negative blood cultures, independently from the etiopathogenesis of sepsis

Table II. Serum level of Vascular endothelial growth factor C (VEGF-C) in the examined groups

\begin{tabular}{cccccc}
\hline \multicolumn{1}{l}{ Number of animals } & Median $(\mathbf{p g} / \mathbf{m l})$ & Minimum (pg/ml) & Maximum (pg/ml) & Interquartile range \\
\hline Group A & 8 & 89.46 & 39.05 & 188.0 & 100.27 \\
\hline & 4 (Blood culture +) & 64.82 & 39.05 & 93.99 & 47.57 \\
\hline & 4 (Blood culture -) & 134.6 & 84.92 & 188.0 & 57.37 \\
\hline Group B & 8 & 71.50 & 50.56 & 99.19 & 34.26 \\
\hline Group C & 6 & 53.65 & 41.76 & 66.65 & 16.24 \\
\hline
\end{tabular}

The horizontal line on the box-plot in figure 1 represents the median value. The length of the box represents the interquartile range (IQR), the upper limit of the box-plot being the $75^{\text {th }}$ percentile, and the inferior limit the $25^{\text {th }}$ percentile. The horizontal lines extending from the ends of the box-plots represent the maximal and minimal serum VEGF-C values. A statistically significant difference was observed between the median values of the three groups $(p=0.04)$.

\section{Immunohistochemical assessment of PDPN in tissues}

\section{Lung}

In all of the cases, PDPN was expressed by the endothelial cells of blood and lymphatic vessels, and by the alveolocytes. As fewer than $50 \%$ of alveolocytes only slightly expressed PDPN (1+), in Group B and C, these specimens were considered negative for PDPN. In contrast, a high score $(3+)$ was proved in cases from group A, in alveolocytes, without differences between those with positive- vs. negative blood cultures (Figure 2, Table III).

\section{Liver}

In liver tissue samples, PDPN expression was positive in all groups, with a distribution score of 2 , meaning that more than $50 \%$ of the hepatocytes slightly expressed PDPN. The expression intensity was rather moderate for Group A (total score 3, sum 4), and weak for Groups B and C (total score 2, sum 3). The expression pattern was relatively specific, being more intense within the portal spaces, compared with the centrilobular veins areas (Table 3). In Group A and B, inflammatory cells were also present and marked by 


\section{Group A \\ E. coli live bacteria - in- duced sepsis}

\section{Group B \\ Ultrapure li- popolysacha- ride from Heat-Killed E. coli - in- duced sepsis}

\section{Group C}

Control group
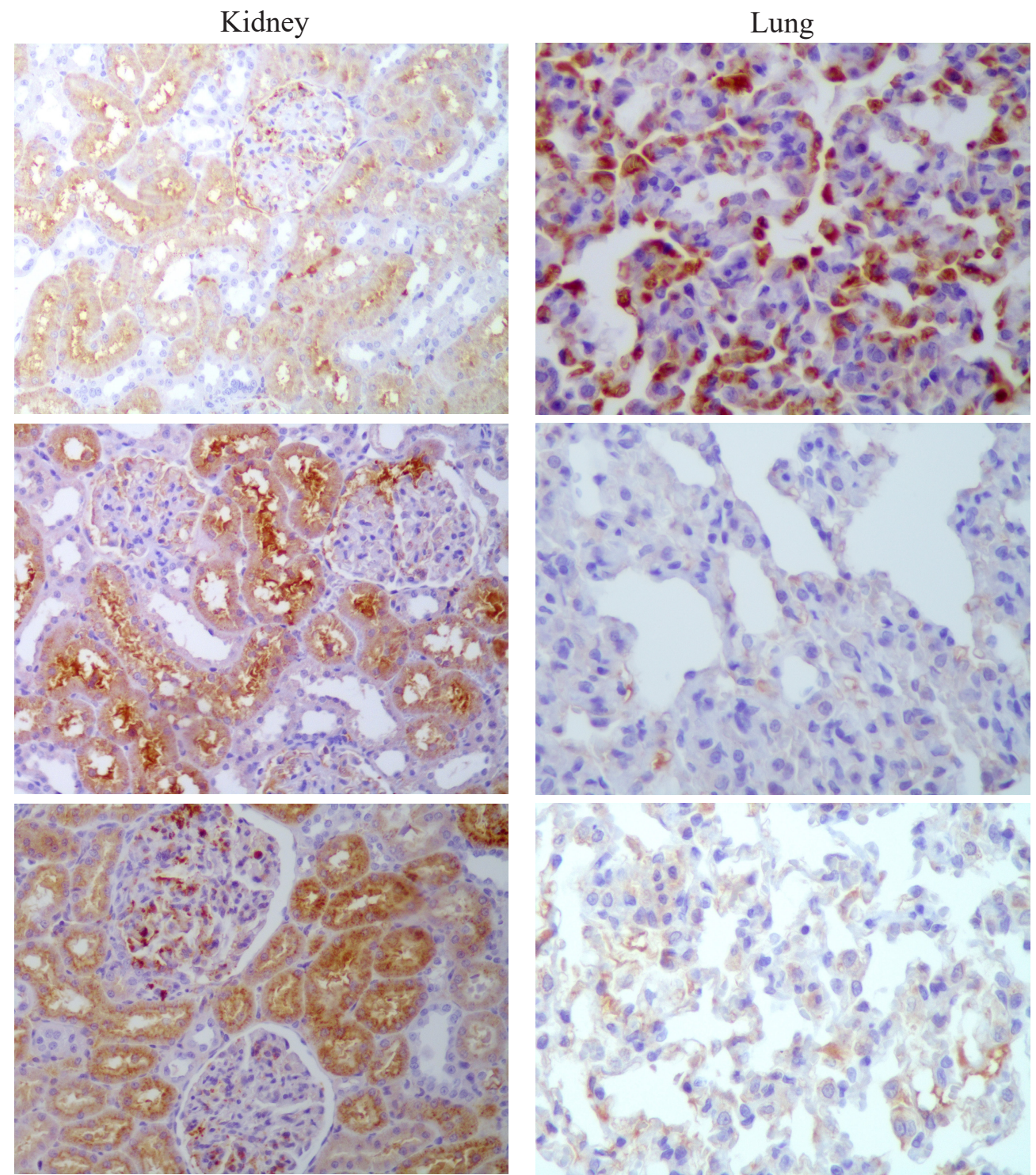

Fig. 2. Immunohistochemical expression of podoplanin. In group A, podoplanin is intensified in the alveolocytes and downregulated in the tubular epithelium of the kidney. In group B, podoplanin expression is kept in tubules and not upregulated in alveolocytes.

PDPN. It also marked inflammatory cells from lymph nodes and spleen but the results were not of interst (data not shown).

\section{Kidney}

In the glomeruli, the PDPN expression was identified in the podocytes and the urinary pole of the
Bowman's capsule. In the tubular system, it was expressed in the proximal tubules and in the endothelium of interstitial vessels. The epithelium of the distal tubules and the collecting ducts was not marked by PDPN. In all groups, PDPN was expressed by more than $50 \%$ of the cells (distribution score 2). In Group A, the intensity was 
Table III. Immunohistochemistry score of podoplanin (PDPN) expression in the examined organs

\begin{tabular}{llccccc}
\hline \multirow{3}{*}{ Lung } & & DS & IS & Sum & TS & Expression \\
\hline \multirow{3}{*}{ Liver } & Group A & 1 & 3 & 4 & 3 & Positive \\
\cline { 2 - 7 } & Group B & 1 & 1 & 2 & 1 & Negative \\
\cline { 2 - 7 } & Group C & 1 & 1 & 2 & 1 & Negative \\
\hline \multirow{3}{*}{ Kidney } & Group A & 2 & 2 & 4 & 3 & Positive \\
\cline { 2 - 7 } & Group B & 2 & 1 & 3 & 2 & Positive \\
\cline { 2 - 6 } & Group C & 2 & 1 & 3 & 2 & Positive \\
\cline { 2 - 6 } & Group A & 2 & 2 & 4 & 3 & Positive \\
\cline { 2 - 6 } & Group B & 2 & 3 & 5 & 3 & Positive \\
\cline { 2 - 6 } & Group C & 2 & 3 & 5 & 3 & Positive \\
\hline
\end{tabular}

moderate to weak (score 2), while in Group B and $\mathrm{C}$ a higher intensity was seen in tubular epithelium (intensity score 3); thus, the expression was positive for all groups (Total score 3 , sum 4 in Group A; Total score 3 and sum 5 for Groups $\mathrm{B}$ and $\mathrm{C}$ ). An intensification of the expression in the apical border of the proximal tubule cells was also observed (Figure 2, Table 3).

DS distribution score: $0(0 \%), 1(1 \%-50 \%)$, or 2 $(51 \%-100 \%)$, reflecting the percentage of positive-stained cells present in the same tissue core; IS - intensity score reflecting the intensity of the signal: 0 (no signal), 1 (weak signal), 2 (moderate signal), and 3 (marked signal); TS - total score is the sum of distribution and intensity score: $\mathrm{TS}=0$ (sum, 0), TS $=1$ (sum, 2), TS $=2$ (sum, 3), and TS = 3 (sum, 4 or 5); Expression: TS of 0 and 1 are considered negative, and TS of 2 and 3 are considered positive.

\section{Discussion}

The new definition of sepsis underscores the involvement of multiple organ systems precipitated by an inadequate immune response to bacterial endotoxins (LPS). This provides us with a new opportunity to study cellular and humoral changes associated with sepsis and the evaluation of biomarkers, which may aid in an early and more objective established diagnosis of sepsis, with further possibility of improving therapeutic management of such cases (24).
The fact that serum VEGF-C levels did not statistically differ between Groups A and B, could be attributed to the higher coefficient of variation for group A than group B. When group A was splitted in cases with positive vs. negative blood cultures, a similar high level, such in group B, was proved for negative $v s$. positive cases.

It was previously shown that LPS potently stimulates VEGF expression in animal models via the cytokine response (25). During septic shock, LPS is released in the infection site following bacterial lysis, and transported in the circulatory system in a complex with the LPS-binding protein (26). LPS is a conserved surface component of all gram-negative bacteria, including E. coli, with virtually no differences regarding the immune signaling. Lipid A is a component of LPS, highly conserved at species level, which is recognized in very small amounts by the innate immune system (TLR4 receptor) and triggers the release of proinflammatory cytokines (27). The core oligosaccharide and the $\mathrm{O}$ antigen are the other two components of LPS, which vary at species and strain level, and are not required for the immunostimulatory activity of LPS. The $\mathrm{O}$ antigen contributes to the pathogen's immune system evasion mechanisms, rather than being involved in inflammation and sepsis evolution $(28,29)$.

Some animals, including rodents, are naturally more resistant than humans to the activity of 
LPS, thus needing high doses in order to trigger an inflammatory response. In our model, the highest possible dose was used (LPS corresponding to $\left.10^{7} \mathrm{CFU} / \mathrm{ml}\right)$. Similarly, a high dose of live bacteria $\left(1.5 \times 10^{8} \mathrm{CFU} / \mathrm{ml}\right)$ was used to induce sepsis, due to the resilience of rodents to bacterial invasion (30).

In line to our data, Guangwei et al demonstrated that LPS can increase VEGF-C secretion which is followed by activation of NF- $\mathrm{BB}$ in macrophages, promoting cell motility and lymphangiogenesis (31). Similar data were reported in mouse models of gram negative septic shock (32).

Based on the common role of VEGF-C and PDPN in lymphangiogenesis, we hypothesized that the serum level of circulating VEGF-C might be correlated with tissue activity of PDPN (31). In septic shock, early renal impairment occurs in up to $60 \%$ of patients, therefore the survival rate might depend on the integrity of the PDPN-positive podocytes (as the control group showed overexpressed PDPN) (33-35). Moreover, it is well-known that the inflammatory response, that occurs in acute kidney injury (AKI), secondary to sepsis or septic shock, damages tubular epithelial cells, followed by significant necrosis observed in the proximal tubule (33). As consequence, hypoxia especially affect the proximal tubules which, in our histological assessment, proved to secrete PDPN.

As the highest VEGF-C serum was found in group A with positive blood culture but PDPN expression was lower in proximal tubules in group A compared to Group B, we conclude that high circulating VEGF-C might reflect LPSinduced damage of proximal tubules as well as podocytes. In one study it was shown that PDPN expression in the podocytes does not alter the LPS induced AKI (22).

Ugorski et al demonstrated that PDPN is expressed by type-I pneumocytes, having a crucial role in lung development, regulating the proliferative potential of the cells thus type-I pneumo- cyte maturation (36). Furthermore, PDPN null mice die shortly after birth, due to respiratory failure (37). Our results were not in agreement with these findings, since the PDPN expression in the lungs of the control group was weeak or negative. As cases from group A highly expressed PDPN in alveolocytes, it can be supposed that it exerts a protective role against lung injury in sepsis induced by live bacteria.

In liver, hepatocytes and subendothelial walls can express PDPN but the clinical significance is not clear (38). However, PDPN is markedly upregulated in LPS stimulation compared to live bacterial injections (39).

A limitation of our study is that the negative blood cultures we obtained could possibly be attributed to the isolate of E.Coli which was done according to predefined standards by Vitek2 Compact automated system (bioMérieux SA, France) and in the ROSCO Neo-Sensitabs tablet synergy tests (Rosco Diagnostica, Denmark). Another limitation refers to lack of evidences to determine the stages and severity of sepsis.

\section{Conclusions}

Serum VEGF-C could be used as a potential biomarker in proinflammatory stages of sepsis and as a predictor for AKI development in septic shock. PDPN is highly expressed in kidney, in LPS-induced sepsis, and in lung, in bacteria-induced sepsis. The clinical potential of these findings need to be checked by further experiments.

\section{Acknowledgments}

The English proofread was done by Cambridge Proofreading and Editing Services. We acknowledge Genoveva Rigmanyi for performing immunohistochemical stains.

\section{Conflicts of Interest}

The authors declare no conflict of interest. 


\section{References}

1. Adhikari NKJ, Fowler RA, Bhagwanjee S, Rubenfeld GD. Critical care and the global burden of critical illness in adults. Lancet. 2010 Oct 16;376(9749):133946. DOI: 10.1016/S0140-6736(10)60446-1

2. Fleischmann C, Scherag A, Adhikari NKJ, Hartog CS, Tsaganos T, Schlattmann P, et al. Assessment of Global Incidence and Mortality of Hospital-treated Sepsis. Current Estimates and Limitations. Am J Respir Crit Care Med. 2016 Feb 1;193(3):259-72. DOI: 10.1164/ rccm.201504-07810C

3. Minasyan H. Sepsis and septic shock: Pathogenesis and treatment perspectives. J Crit Care. 2017 Aug;40:22942. DOI: 10.1016/j.jcrc.2017.04.015

4. Sherwin R, Winters ME, Vilke GM, Wardi G. Does Early and Appropriate Antibiotic Administration Improve Mortality in Emergency Department $\mathrm{Pa}$ tients with Severe Sepsis or Septic Shock? J Emerg Med. 2017 Oct;53(4):588-95. DOI: 10.1016/j. jemermed.2016.12.009

5. Rhee C, Dantes R, Epstein L, Murphy DJ, Seymour CW, Iwashyna TJ, et al. Incidence and Trends of Sepsis in US Hospitals Using Clinical vs Claims Data, 2009-2014. JAMA. 2017 Oct 3;318(13):1241-9. DOI: 10.1001/jama.2017.13836

6. Giannakopoulos K, Hoffmann U, Ansari U, Bertsch T, Borggrefe M, Akin I, et al. The Use of Biomarkers in Sepsis: A Systematic Review. Curr Pharm Biotechnol. 2017;18(6):499-507. DOI: 10.2174/138920101866617 0601080111

7. Kovacs J, Gurzu S, Jung J, Szederjesi J, Copotoiu S-M, Copotoiu R, et al. Clinico-pathological particularities of the shock-related pancreatitis. Pathol Oncol Res. 2012 Oct;18(4):977-81. DOI: 10.1007/s12253-0129528-6

8. Gauer R, Forbes D, Boyer N. Sepsis: Diagnosis and Management. AFP. 2020 Apr 1;101(7):409-18.

9. Matthaiou DK, Ntani G, Kontogiorgi M, Poulakou G, Armaganidis A, Dimopoulos G. An ESICM systematic review and meta-analysis of procalcitonin-guided antibiotic therapy algorithms in adult critically ill patients. Intensive Care Med. 2012 Jun;38(6):940-9. DOI: 10.1007/s00134-012-2563-7

10. Wacker C, Prkno A, Brunkhorst FM, Schlattmann P. Procalcitonin as a diagnostic marker for sepsis: a systematic review and meta-analysis. Lancet Infect
Dis. 2013 May;13(5):426-35. DOI: 10.1016/S14733099(12)70323-7

11. Lam SW, Bauer SR, Fowler R, Duggal A. Systematic Review and Meta-Analysis of Procalcitonin-Guidance Versus Usual Care for Antimicrobial Management in Critically Ill Patients: Focus on Subgroups Based on Antibiotic Initiation, Cessation, or Mixed Strategies. Crit Care Med. 2018 May;46(5):684-90. DOI: 10.1097/ CCM.0000000000002953

12. Karaman S, Leppänen V-M, Alitalo K. Vascular endothelial growth factor signaling in development and disease. Development. 2018 Jul 20;145(14):dev151019. DOI: $10.1242 /$ dev.151019

13. Park JE, Chen HH, Winer J, Houck KA, Ferrara N. Placenta growth factor. Potentiation of vascular endothelial growth factor bioactivity, in vitro and in vivo, and high affinity binding to Flt-1 but not to Flk-1/KDR. J Biol Chem. 1994 Oct 14;269(41):25646-54. DOI: 10.1016/S0021-9258(18)47298-5

14. Joukov V, Sorsa T, Kumar V, Jeltsch M, Claesson-Welsh $\mathrm{L}$, Cao Y, et al. Proteolytic processing regulates receptor specificity and activity of VEGF-C. EMBO J. 1997 Jul 1;16(13):3898-911. DOI: 10.1093/emboj/16.13.3898

15. Maglione D, Guerriero V, Viglietto G, Delli-Bovi P, Persico MG. Isolation of a human placenta cDNA coding for a protein related to the vascular permeability factor. Proc Natl Acad Sci U S A. 1991 Oct 15;88(20):926771. DOI: $10.1073 /$ pnas.88.20.9267

16. Azamfirei L, Gurzu S, Solomon R, Copotoiu R, Copotoiu S, Jung I, et al. Vascular endothelial growth factor: a possible mediator of endothelial activation in acute respiratory distress syndrome. Minerva Anestesiol. 2010 Aug;76(8):609-16.

17. Astarita JL, Acton SE, Turley SJ. Podoplanin: emerging functions in development, the immune system, and cancer. Front Immunol. 2012;3:283. DOI: 10.3389/fimmu.2012.00283

18. Wicki A, Christofori G. The potential role of podoplanin in tumour invasion. Br J Cancer. 2007 Jan 15;96(1):1-5. DOI: $10.1038 /$ sj.bjc. 6603518

19. Suzuki-Inoue K, Inoue O, Ozaki Y. Novel platelet activation receptor CLEC-2: from discovery to prospects. J Thromb Haemost. 2011 Jul;9 Suppl 1:44-55. DOI: 10.1111/j.1538-7836.2011.04335.x

20. Breiteneder-Geleff S, Matsui K, Soleiman A, Meraner P, Poczewski H, Kalt R, et al. Podoplanin, novel 43$\mathrm{kd}$ membrane protein of glomerular epithelial cells, is 
down-regulated in puromycin nephrosis. Am J Pathol. 1997 Oct;151(4):1141-52.

21. Maruyama Y, Maruyama K, Kato Y, Kajiya K, Moritoh S, Yamamoto K, et al. The effect of podoplanin inhibition on lymphangiogenesis under pathological conditions. Invest Ophthalmol Vis Sci. 2014 Jul 1;55(8):4813-22. DOI: 10.1167/iovs.13-13711

22. Rayes J, Lax S, Wichaiyo S, Watson SK, Di Y, Lombard $\mathrm{S}$, et al. The podoplanin-CLEC-2 axis inhibits inflammation in sepsis. Nat Commun. 2017 Dec 21;8(1):2239 DOI: $10.1038 / \mathrm{s} 41467-017-02402-6$

23. Kitano H, Kageyama S-I, Hewitt SM, Hayashi R, Doki Y, Ozaki Y, et al. Podoplanin expression in cancerous stroma induces lymphangiogenesis and predicts lymphatic spread and patient survival. Arch Pathol Lab Med. 2010 Oct;134(10):1520-7. DOI: 10.5858/20090114-OA.1

24. Singer M, Deutschman CS, Seymour CW, Shankar-Hari M, Annane D, Bauer M, et al. The Third International Consensus Definitions for Sepsis and Septic Shock (Sepsis-3). JAMA. 2016 Feb 23;315(8):801-10. DOI: $10.1001 /$ jama.2016.0287

25. Hahn RG. Endotoxin boosts the vascular endothelial growth factor (VEGF) in rabbits. J Endotoxin Res. 2003;9(2):97-100. DOI: 10.1177/09680519030090020401

26. Adamik B, Zielinski S, Smiechowicz J, Kübler A. Endotoxin Elimination in Patients with Septic Shock: An Observation Study. Arch Immunol Ther Exp (Warsz). 2015 Dec;63(6):475-83. DOI: 10.1007/s00005-0150348-8

27. Ramachandran G. Gram-positive and gram-negative bacterial toxins in sepsis: a brief review. Virulence. 2014 Jan 1;5(1):213-8. DOI: 10.4161/viru.27024

28. Bertani B, Ruiz N. Function and Biogenesis of Lipopolysaccharides. EcoSal Plus. 2018 Aug;8(1):10.1128/ ecosalplus.ESP-0001-2018. DOI: 10.1128/ecosalplus. ESP-0001-2018

29. Matsuura M. Structural Modifications of Bacterial Lipopolysaccharide that Facilitate Gram-Negative Bacteria Evasion of Host Innate Immunity. Front Immunol. 2013;4:109. DOI: 10.3389/fimmu.2013.00109

30. Poli-de-Figueiredo LF, Garrido AG, Nakagawa N, Sannomiya P. Experimental models of sepsis and their clinical relevance. Shock. 2008 Oct;30 Suppl 1:53-9. DOI:
10.1097/SHK.0b013e318181a343

31. Zhu G, Huang Q, Huang Y, Zheng W, Hua J, Yang S, et al. Lipopolysaccharide increases the release of VEGF-C that enhances cell motility and promotes lymphangiogenesis and lymphatic metastasis through the TLR4NF- $\mathrm{KB} / \mathrm{JNK}$ pathways in colorectal cancer. Oncotarget. 2016 Oct 4;7(45):73711-24. DOI: 10.18632/oncotarget.12449

32. Zhang Y, Lu Y, Ma L, Cao X, Xiao J, Chen J, et al. Activation of vascular endothelial growth factor receptor-3 in macrophages restrains TLR4-NF- $\mathrm{kB}$ signaling and protects against endotoxin shock. Immunity. $2014 \mathrm{Apr}$ 17;40(4):501-14. DOI: 10.1016/j.immuni.2014.01.013

33. Pan P, Liu X, Wu L, Li X, Wang K, Wang X, et al. TREM-1 promoted apoptosis and inhibited autophagy in LPS-treated HK-2 cells through the NF- $\mathrm{kB}$ pathway. Int J Med Sci. 2021 Jan 1;18(1):8-17. DOI: 10.7150/ ijms.50893

34. Foster RR, Satchell SC, Seckley J, Emmett MS, Joory $\mathrm{K}$, Xing CY, et al. VEGF-C promotes survival in podocytes. Am J Physiol Renal Physiol. $2006 \mathrm{Ju}-$ 1;291(1):F196-207. DOI: 10.1152/ajprenal.00431.2005

35. Quintanilla M, Montero-Montero L, Renart J, Martín-Villar E. Podoplanin in Inflammation and Cancer. Int J Mol Sci. 2019 Feb 6;20(3):707. DOI: 10.3390/ ijms20030707

36. Ugorski M, Dziegiel P, Suchanski J. Podoplanin - a small glycoprotein with many faces. Am J Cancer Res. 2016;6(2):370-86.

37. Winde CM de, Makris S, Millward L, Benjamin AC, Cazzagon G, Martinez VG, et al. Podoplanin function is switched by partner proteins on fibroblastic reticular cells. BioRxiv. 2019 Nov (cited 2021 Sep 21):793141. DOI: $10.1101 / 793141$

38. Hitchcock JR, Cook CN, Bobat S, Ross EA, Flores-Langarica A, Lowe KL, et al. Inflammation drives thrombosis after Salmonella infection via CLEC-2 on platelets. J Clin Invest. 2015 Dec;125(12):4429-46. DOI: 10.1172/JCI79070

39. Kerrigan AM, Navarro-Nu-ez L, Pyz E, Finney BA, Willment JA, Watson SP, et al. Podoplanin-expressing inflammatory macrophages activate murine platelets via CLEC-2. J Thromb Haemost. 2012 Mar;10(3):4846. DOI: $10.1111 / j .1538-7836.2011 .04614 . x$ 